vol. $20-n^{\circ} 1 \mid 2004$

Numéro ouvert

\title{
Les entrées d'étrangers sur le marché du travail français (1990-2001)
}

Jean-François Léger

\section{(2) OpenEdition}

1 Journals

Édition électronique

URL : https://journals.openedition.org/remi/261

DOI : $10.4000 /$ remi.261

ISSN : $1777-5418$

Éditeur

Université de Poitiers

Édition imprimée

Date de publication : 1 mai 2004

Pagination : 7-31

ISBN : 2-911627-36-9

ISSN : 0765-0752

Référence électronique

Jean-François Léger, «Les entrées d'étrangers sur le marché du travail français (1990-2001) », Revue européenne des migrations internationales [En ligne], vol. 20 - $\mathrm{n}^{\circ} 1$ | 2004, mis en ligne le 24 septembre 2008, consulté le 14 avril 2022. URL : http://journals.openedition.org/remi/261 ; DOI : https://doi.org/ 10.4000/remi.261

Ce document a été généré automatiquement le 14 avril 2022.

() Université de Poitiers 


\title{
Les entrées d'étrangers sur le marché du travail français (1990-2001)
}

\author{
Jean-François Léger
}

1 Chaque année, quelques milliers d'étrangers entrent en France afin d'exercer une activité professionnelle (salariée ou non). Ils ne constituent qu'une partie du flux annuel d'entrées d'étrangers sur le marché du travail français. En effet, parmi les étrangers qui entrent légalement en France à un autre titre que l'exercice d'une activité professionnelle pour une durée au moins égale à un an, certains deviennent actifs l'année même de leur arrivée en France. D'autres, enfin, diffèrent leur accès au marché de l'emploi les années suivant leur entrée dans l'hexagone.

Trois catégories d'entrées sur le marché du travail peuvent donc être définies :

- - les « entrées directes » : il s'agit des étrangers qui entrent en France une année donnée afin d'exercer une activité salariée ou non ;

- - les « entrées indirectes » : elles correspondent aux étrangers qui entrent sur le marché du travail l'année même de leur arrivée en France à un autre titre que l'exercice d'une activité. Les actifs potentiels sont les étrangers âgés de plus de 16 ans qui bénéficient de la procédure du regroupement familial, des conjoints et parents de Français, des réfugiés politiques et des membres de familles de réfugiés ;

- les « entrées différées » : il s'agit des étrangers présents sur le territoire français le 1er janvier de l'année considérée, qui ne sont encore jamais entrés sur le marché du travail et qui deviennent précisément actifs pour la première fois au cours de cette même année.

Depuis la mise en place de titres uniques autorisant à la fois de séjourner en France et d'y travailler, institués par la loi n 84-622 du 17 juillet 1984, seules les «entrées directes » sont recensées. Les entrées « indirectes » et "différées » ne font pour leur part l'objet d'aucun dénombrement. C'est précisément pour combler ce vide statistique, que la Direction de la population et des migrations (DPM) a initié en 1996 une série d'études destinées à produire une estimation du nombre d'étrangers qui entrent chaque année sur le marché du travail français. En 2003 a ainsi été réalisée la quatrième 
étude sur ce thème, consacrée aux années 1999-2001 (Léger, 2003)1․ Les résultats qui en sont issus viennent compléter la série statistique qui couvrait jusqu'à présent les années 1990-1998.

Dans le cadre de cette étude, seules sont considérées les entrées sur le marché du travail d'étrangers qui bénéficient d'une autorisation de séjour en France valide pour une durée au moins égale à un an, à l'exception, d'une part, des étudiants en raison de leur état par définition provisoire et des conditions réglementaires particulières qui régissent leur possibilité d'exercer une activité et, d'autre part, des visiteurs, puisque l'ordonnance de 1945 prévoit explicitement que l'engagement de n'exercer en France aucune activité professionnelle soumise à autorisation est l'une des conditions exigées pour la délivrance de ce titre. Les travailleurs temporaires tels que les bénéficiaires d'une autorisation provisoire de travail (APT) ou les stagiaires, les travailleurs saisonniers et, bien sûr, les étrangers en situation irrégulière échappent aussi à notre champ d'observation. Ce choix permet en outre de rapprocher le flux annuel d'entrées de nouveaux actifs étrangers "permanents» sur le marché de l'emploi français du nombre global de personnes qui, chaque année, en France, entrent pour la première fois sur le marché du travail.

\section{Les « entrées directes »}

\section{Près de 23000 « entrées directes » en 2001}

5 L'effectif des "entrées directes » est connu avec une bonne précision. En effet, la plupart des étrangers qui entrent en France dans le cadre de cette procédure font l'objet d'un contrôle sanitaire effectué par l'office des Migrations Internationales (OMI). On connait donc bien leur nombre ainsi que leur distribution par sexe et âge. Cependant, certaines nationalités échappent à ce contrôle sanitaire. Il s'agit, jusqu'en 1993, des pays appartenant à l'Union européenne (UE) et, depuis 1994, de l'ensemble des pays, membres ou non de l'Union européenne, composant l'espace économique européen (EEE), dont les ressortissants bénéficient de l'accord de libre circulation des personnes, signé entre ces pays².

6 Pour les études relatives aux années 1990-1998, l'effectif des étrangers originaires des pays de l'EEE a été approché à partir des déclarations d'engagement souscrites par les employeurs pour les actifs salariés. La seule incertitude concernait donc le flux d'actifs non salariés, qui a été estimé sur la base de l'évaluation annuelle proposée par la DPM, qui tenait compte des indications fournies par le ministère de l'intérieur et par l'évolution des flux de salariés. Pour les années 1999-2001, l'estimation des «entrées directes » des étrangers originaires de l'EEE se fonde, pour les actifs salariés et non salariés, sur les résultats directement fournis par l'AGDREF ${ }^{3}$. En effet, il s'est avéré que les statistiques issues de l'AGDREF sont plus complètes que celles de l'OMI. L'OMI recense ainsi un peu plus de 5000 entrées d'actifs salariés originaires de l'EEE en 1999 et 2000 (respectivement 5569 et 5 352), tandis que d'après l'AGDREF, ce flux est deux fois plus important (12 727 en 1999 et 13479 en 2000).

7 Au total, on dénombre plus de 19000 "entrées directes " sur le marché du travail français en 1999 et 2000, et près de 23000 en 2001. Au cours de ces trois années, les caractéristiques démographiques sont stables et comparables à celles observées pour les années précédentes (Léger, 2001). Ainsi, sur la période 1999-2001, quel que soit le 
groupe de nationalités, les hommes sont plus nombreux que les femmes. Ils représentent $65 \%$ des "entrées directes" issues de l'EEE et $72 \%$ de celles en provenance des pays tiers. Dans tous les cas, il s'agit de jeunes actifs : les actifs âgés de moins de 30 ans représentent $43 \%$ des «entrées directes " issues de l'EEE et $47 \%$ de celles en provenance des pays tiers. On peut aussi noter que les hommes sont en moyenne légèrement plus âgés que les femmes:

- - pour les ressortissants de l'EEE, les âges moyens sont respectivement de 34 et 32 ans;

- - pour les étrangers originaires des pays tiers, ils sont de 33 et 31 ans.

\section{Évolution entre 1990 et 2001}

L'adoption des données issues de l'AGDREF pour les étrangers originaires des pays membres de l'EEE à la place des statistiques de l'OMI ne remet pas en cause la structure par âge et sexe des "entrées directes ». Il n'en est pas de même pour leur effectif. D'après les données issues de l'AGDREF, il s'avère depuis 1997 (date de la première publication des effectifs issus de cette source), que le nombre d'« entrées directes » a été nettement sous-estimé par l'OMI (graphique 1). Les écarts observés de 1997 à 2000 entre ces deux sources statistiques incitent à réestimer les " entrées directes » entre 1990 et 1996. L'observation des deux courbes montre deux évolutions opposées : alors que les «entrées directes» de l'EEE tendent à augmenter de 1997 à 2001 selon les données issues de l'AGDREF, elles tendent au contraire à diminuer selon l'OMI. Cette baisse peut être attribuée à une diminution régulière du nombre de déclarations d'engagement effectuées (à leur propre initiative) par les employeurs auprès de l'OMI, tandis que l'évolution positive révélée par les données de l'AGDREF traduit une réelle augmentation de ce flux. À partir des écarts entre ces deux courbes, qui diminuent linéairement à mesure que l'on remonte dans le temps, une nouvelle estimation des « entrées directes » est proposée pour les années 1990-19964 (graphique 2). Elle conduit à une série chronologique pour l'ensemble des "entrées directes" depuis 1990, réactualisée par rapport aux chiffres présentés dans nos précédents travaux (Léger, 2001) (tableau 1). 
Graphique 1 : Effectifs d'étrangers originaires des pays membres de l'EEE qui sont entrés en France afin d'exercer une activité professionnelle entre 1990 et 2001 , selon la source statistique

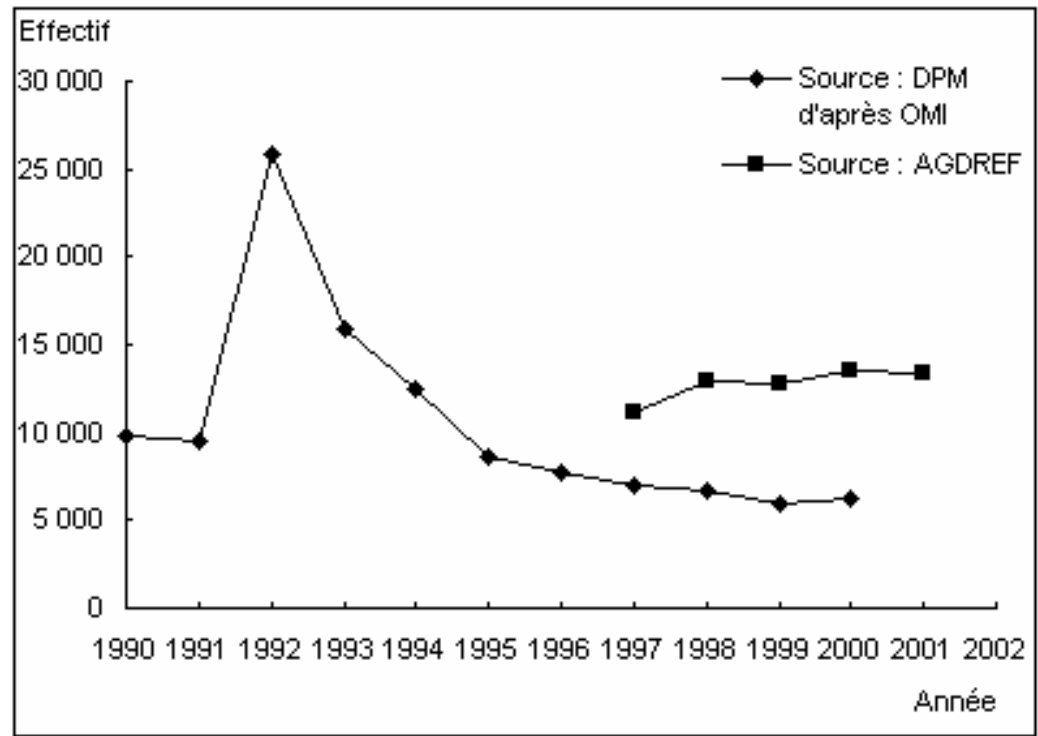

Source : AGDREF, DPM, OMI

Graphique 2 : Estimation des « entrées directes » des étrangers originaires des pays membres de I'EEE de 1990 à 1996

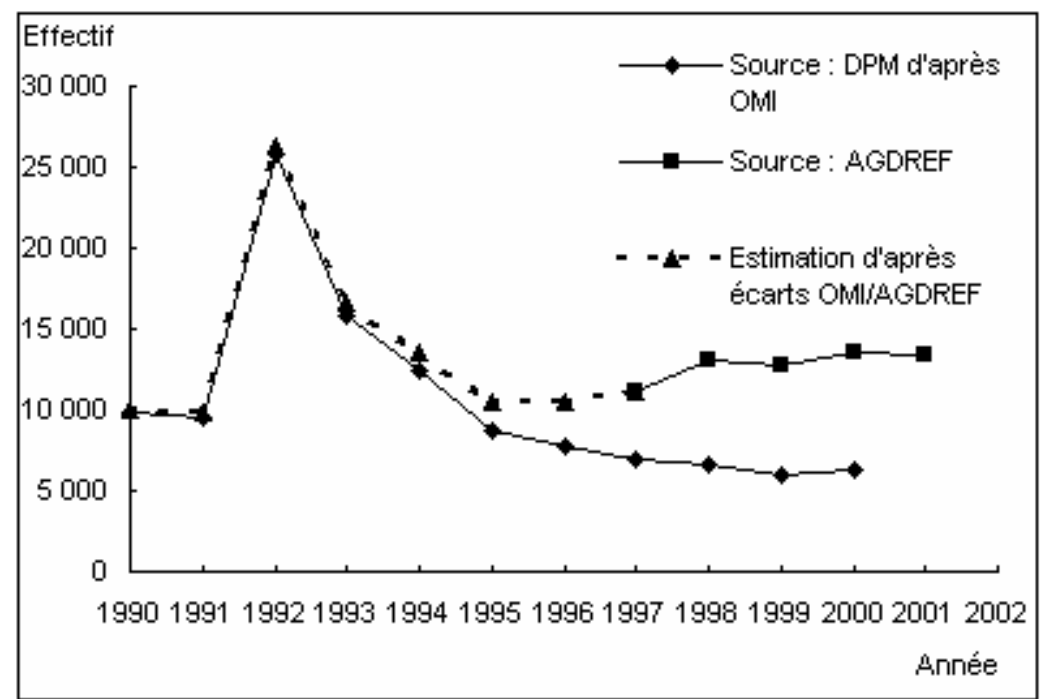


Tableau 1 : Les « entrées directes » de 1990 à 2001 selon le groupe de nationalités et le sexe

\begin{tabular}{|c|c|c|c|c|c|}
\hline \multirow{2}{*}{ Année } & \multicolumn{2}{|c|}{ EEE } & \multicolumn{2}{c|}{ Pays tiers } & \multirow{2}{*}{ Total } \\
\cline { 2 - 5 } & Hommes & Femmes & Hommes & Femmes & \\
\hline 1990 & 6000 & 4000 & 14301 & 4820 & 29121 \\
\hline 1991 & 6000 & 4000 & 16473 & 5553 & 32026 \\
\hline 1992 & 16000 & 10000 & 19708 & 6645 & 52353 \\
\hline 1993 & 10000 & 6500 & 8057 & 3750 & 28307 \\
\hline 1994 & 8000 & 5500 & 5885 & 2343 & 21728 \\
\hline 1995 & 6500 & 4000 & 4340 & 1805 & 16645 \\
\hline 1996 & 6500 & 4000 & 3260 & 1493 & 15253 \\
\hline 1997 & 6729 & 4366 & 3592 & 1645 & 16332 \\
\hline 1998 & 7881 & 5113 & 3723 & 1704 & 18421 \\
\hline 1999 & 8187 & 4540 & 4441 & 1839 & 19007 \\
\hline 2000 & 8911 & 4567 & 4617 & 1786 & 19881 \\
\hline 2001 & 8788 & 4627 & 6641 & 2603 & 22659 \\
\hline
\end{tabular}

Sources : OMI seul pour les actifs ressortissants des pays tiers; AGDREF seule pour les étrangers originaires de l'EEE pour les années 1997-2001; estimation rétrospective pour les étrangers originaires de l'EEE pour les années 1990-1996 à partir des données de l'OMI (corrigées par la DPM) et des écarts observés au cours des années 1997-2001 entre l'OMI et l'AGDREF.

9 Si l'on excepte l'année 1992 au cours de laquelle, d'une part, l'élargissement de la libre circulation aux ressortissants espagnols et portugais et, d'autre part, la « régularisation» de demandeurs d'asile déboutés, avaient entraîné des entrées massives, pour partie réelle, pour partie de nature statistique, dans la catégorie des « entrées directes » (Lebon, 1993), le nombre annuel d'« entrées directes » n'a cessé de décroître entre 1990 et 1996, passant de 30000 à environ à 15 000. Depuis 1997, il tend à remonter, passant progressivement en cinq ans de 16000 entrées annuelles (en 1997) à près de 23000 en 2001. L'évolution est sensiblement différente d'un groupe de nationalités à un autre. Alors que les étrangers originaires des pays tiers étaient deux fois plus nombreux que les ressortissants de l'EEE en 1990, ils sont minoritaires depuis 1993. Au cours des années 1995-2000, ils sont même deux fois moins nombreux que les ressortissants de l'EEE, soit la situation inverse de celle, constatée en 1990. En 2001, cet écart s'est atténué, mais les étrangers en provenance des pays tiers ne représentent que 40 \% du flux d'« entrées directes » recensées cette année-là, contre 66 \% en 1990.

\section{Les « entrées indirectes »}

\section{Quelques précisions méthodologiques}

10 En plus des étrangers qui entrent chaque année en France afin d'exercer une activité, d'autres étrangers, venus dans le cadre d'une autre procédure, sont susceptibles d'accéder au marché du travail l'année même de leur arrivée dans l'hexagone. Il s'agit, dans le cadre de cette étude où seule l'immigration légale à caractère permanent est considérée, des personnes qui bénéficient du regroupement familial, des parents de Français, des réfugiés et des membres de leur famille. À partir de 1999, les titulaires de la carte de séjour «vie privée et familiale » (VPF) ont été inclus dans le champ de l'estimation des « entrées indirectes ». Celle-ci est réalisée en articulant des statistiques relatives, d'une part, aux flux d'entrées sur le territoire français et, d'autre part, à la situation d'activité des étrangers présents en France. En effet, c'est en multipliant les 
taux d'activité par âge, sexe et nationalité regroupée par les effectifs d'actifs potentiels correspondants, que l'on évalue le nombre d'« entrées indirectes » sur le marché du travail.

Cette articulation repose sur l'hypothèse que les étrangers, qui entrent en France à un autre titre que l'exercice d'une activité, vont l'année même de leur arrivée en France, s'inscrire sur le marché du travail dans les mêmes proportions que les étrangers déjà présents en France et présentant les mêmes caractéristiques démographiques (groupe d'âges, nationalité regroupée, sexe). Seule cette hypothèse réduit la précision de l'estimation des «entrées indirectes ». En effet, grâce aux dénombrements effectués par l'OMI (pour les bénéficiaires du regroupement familial, les parents de Français, les membres de familles de réfugiés et les titulaires de la carte de séjour "vie privée et familiale» originaires des pays tiers), l'AGDREF (pour les bénéficiaires du regroupement familial et les parents de Français originaires de l'EEE) et l'Office français de protection des réfugiés et apatrides (pour les réfugiés), le nombre d'étrangers qui entrent chaque année en France pour une durée au moins égale à un an est connu avec une bonne précision. C'est aussi le cas pour les taux d'activité des étrangers en France, à partir desquels l'intensité d'entrer sur le marché du travail est déterminée, qui sont issus de l'enquête sur l'emploi réalisée tous les ans par l'INSEE (graphique 3).

\section{Graphique 3 : Taux d'activité par groupe d'âge des étrangers selon la nationalité regroupée et le sexe}

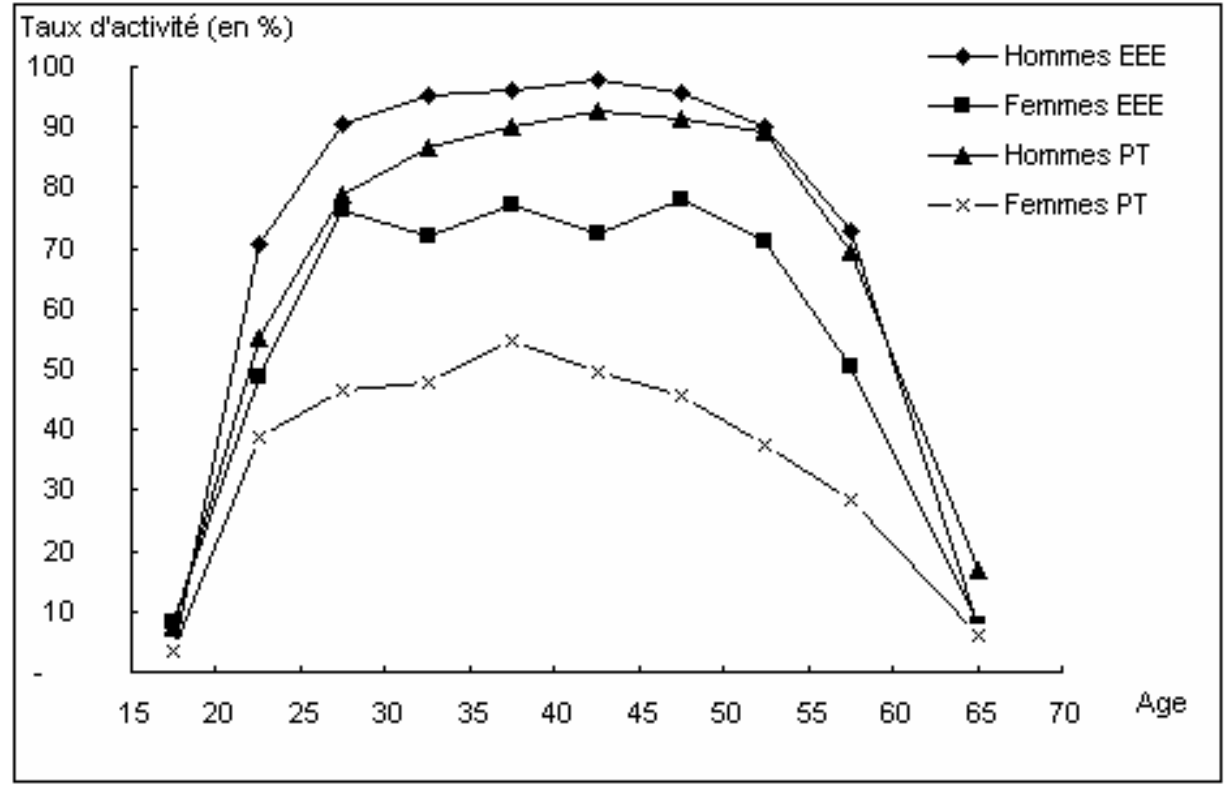

Source : INSEE, enquêtes Emploi 1999, 2000 et 2001.

\section{Le nombre d'actifs potentiels a augmenté depuis 1999}

Le nombre d'étrangers qui sont entrés en France à un autre titre que l'exercice d'une activité professionnelle a considérablement augmenté entre 1999 et 2001 ; ce flux est ainsi passé d'un peu plus de 70000 personnes en 1999 à près de 100000 en 2001 (tableau 2). 
Tableau 2 : Les étrangers qui sont entrés en France à un autre titre que l'exercice d'une activité de 1999 à 2001 selon le type de procédure et la nationalité regroupée

\begin{tabular}{|l|c|c|c|c|c|c|}
\hline \multirow{2}{*}{$\begin{array}{l}\text { Type de } \\
\text { procédure }\end{array}$} & \multicolumn{2}{|c|}{ Année 1999 } & \multicolumn{2}{c|}{ Année 2000 } & \multicolumn{2}{c|}{ Année 2001 } \\
\cline { 2 - 7 } & EEE & Pays tiers & EEE & Pays tiers & EEE & Pays tiers \\
\hline Rgpt familial & 6500 & 21762 & 8750 & 21404 & 8910 & 23081 \\
\hline $\begin{array}{l}\text { Famille de } \\
\text { Français }\end{array}$ & 1700 & 15301 & 2360 & 15992 & 2120 & 18765 \\
\hline Réfugiés & 0 & 4659 & 0 & 5185 & 0 & 7323 \\
\hline $\begin{array}{l}\text { Famille de } \\
\text { Réfugiés }\end{array}$ & 0 & 1379 & 0 & 1700 & 0 & 1999 \\
\hline $\begin{array}{l}\text { Carte de } \\
\text { séjour VPF }\end{array}$ & 0 & 20229 & 0 & 31665 & 0 & 35182 \\
\hline Total & 8200 & 63330 & 11110 & 75946 & 11030 & 86350 \\
\hline
\end{tabular}

Source : AGDREF, OFPRA, OMI, DPM (Lebon, 2001a, 2001b, 2003).

L'importance du nombre d'étrangers originaires des pays tiers recensés dans la rubrique carte de séjour «vie privée et familiale » en fait le premier mode d'entrée en France à un autre titre que l'exercice d'une activité professionnelle. Cette rubrique est composée de nombreux alinéas qui correspondent chacun à un motif particulier d'entrée en France (tableau 3). Afin d'être cohérent avec la ventilation des actifs potentiels adoptée depuis 1990, les détenteurs de la carte de séjour "vie privée et familiale " ont été rapprochés des quatre grands groupes d'actifs potentiels pris en compte dans le cadre de l'estimation des "entrées indirectes" (bénéficiaires du regroupement familial, parents de Français, réfugiés et membres de leur famille). Ainsi :

- les apatrides, les conjoints de scientifiques, les mineurs de moins de 19 ans qui résident en France depuis l'âge de dix ans et les étrangers nés en France, qui y ont résidé pendant huit ans dont cinq ans de scolarité, ont été assimilés à des bénéficiaires du regroupement familial ;

- - les conjoints de Français et les parents d'enfants français mineurs résidant en France ont été inclus parmi les membres de familles de Français ;

- les bénéficiaires de l'asile territorial ont été agrégés aux réfugiés. 
Tableau 3 : Répartition des titulaires de la carte de séjour « vie privée et familiale » selon le motif de délivrance de la carte pour les années 1999-2001

\begin{tabular}{|l|r|r|r|}
\hline Motif de délivrance & 1999 & 2000 & 2001 \\
\hline Apatride ou conjoint ou enfant <= à 18 ans & 14 & 20 & 23 \\
\hline Asile territorial ou conjoint ou enfant <= à 18 ans (1) & 642 & 910 & 822 \\
\hline Conjoint de Français & 10499 & 17081 & 20244 \\
\hline Conjoint de scientifique & 181 & 334 & 366 \\
\hline Liens personnels et familiaux & 3314 & 5093 & 5564 \\
\hline Mineur <= à 18 ans, résidence habituelle depuis l'âge de 10 ans & 1602 & 2306 & 1853 \\
\hline Né en France, résidence pendant 8 ans, dont scolarité pendant 5 ans & 48 & 65 & 45 \\
\hline Parent d'enfant français mineur résidant en France & 1596 & 2939 & 3558 \\
\hline Rente >= à 20 \% & 7 & 8 & 8 \\
\hline $\begin{array}{l}\text { Résidence habituelle depuis + de 10 ans ou + de 15 ans en qualité } \\
\text { d'étudiant }\end{array}$ & 2326 & 2909 & 2699 \\
\hline Total & 20229 & 31665 & 35182 \\
\hline
\end{tabular}

Source : OMI, DPM (Lebon, 2001a, 2001b, 2003).

(1) Dont 350 estimés par la DPM en 1999, 525 en 2000 et 500 en 2001 (Lebon, 2001a, 2001b, 2003)

14 Les bénéficiaires de la carte de séjour "vie privée et familiale » en raison de «liens personnels et familiaux » ou parce que leur «résidence habituelle depuis plus de dix ans ou plus de quinze ans en qualité d'étudiant » est la France, ont été assimilés à des bénéficiaires du réexamen de 1997 (cf. infra). Enfin, deux catégories de titulaires de la carte « vie privée et familiale » ont été exclues du champ de l'estimation : les étrangers malades et les bénéficiaires d'une rente. Nous avons considéré que l'état de santé des premiers et l'existence de revenus pour les seconds incitaient la plupart de ces étrangers à ne pas entrer sur le marché de l'emploi.

15 La répartition des titulaires de la carte "vie privée et familiale " selon le motif de délivrance de ce titre de séjour, puis l'agrégation de chacun de ces groupes à la catégorie d'actifs potentiels originaires des pays tiers entrés en France à un autre titre que l'exercice d'une activité rémunérée qui lui correspond le mieux, permet de définir une nouvelle distribution des actifs potentiels selon le motif d'entrée en France (tableau 4). 
Tableau 4 : Récapitulatif des effectifs de référence à partir desquels sont dénombrés les actifs potentiels, selon le motif d'entrée en France et la nationalité regroupée

\begin{tabular}{|l|c|r|r|r|r|r|}
\hline \multirow{2}{*}{$\begin{array}{l}\text { Motif d'entrée en } \\
\text { France }\end{array}$} & \multicolumn{2}{|c|}{ Année 1999 } & \multicolumn{2}{c|}{ Année 2000 } & \multicolumn{2}{c|}{ Année 2001 } \\
\cline { 2 - 7 } & EEE & PT & EEE & PT & EEE & PT \\
\hline $\begin{array}{l}\text { Regroupement } \\
\text { familial }\end{array}$ & 6500 & 23607 & 8750 & 24129 & 8910 & 25368 \\
\hline $\begin{array}{l}\text { Famille de } \\
\text { Français }\end{array}$ & 1700 & 27396 & 2360 & 36012 & 2120 & 42567 \\
\hline Réfugiés & - & 5301 & - & 6095 & & 8145 \\
\hline $\begin{array}{l}\text { Famille de } \\
\text { réfugiés }\end{array}$ & - & 1379 & - & 1700 & & 1999 \\
\hline Total & 8200 & 57683 & 11110 & 67936 & 11030 & 78079 \\
\hline
\end{tabular}

Source : OMI, OFPRA, AGDREF, DPM (Lebon, 2001a, 2001b, 2003)

16 Tous les étrangers entrés en France comme membres de familles de Français ou réfugiés ou membres de familles de réfugiés - dès lors qu'ils sont enregistrés dans les états statistiques - sont majeurs. De ce fait, ils peuvent tous devenir actifs. Ce n'est pas le cas de tous les bénéficiaires du regroupement familial. Parmi les étrangers originaires des pays tiers qui entrent en France dans le cadre de cette procédure (hors "VPF »), on dénombre en effet 8553 enfants âgés de moins de 15 ans en 1999, 7717 en 2000 et 8091 en 2001. Compte tenu de leur âge, ils ne peuvent pas entrer sur le marché du travail. C'est pourquoi, alors qu'on dénombre 23607 bénéficiaires du regroupement familial originaires des pays tiers en 1999 (y compris les titulaires de la carte «vie privée et familiale »), 24129 en 2000 et 25368 en 2001, les actifs potentiels issus de ce flux d'entrée en France ne sont que 15054 en 1999, 16412 en 2000 et 17727 en 2001. De ce fait, le nombre d'actifs potentiels est inférieur à l'effectif d'étrangers qui sont entrés en France à un autre titre que l'exercice d'une activité.

L'effectif de ces actifs potentiels a considérablement augmenté depuis 1999, sous l'effet, principalement, de la création de la carte de séjour « vie privée et familiale ». Il passe ainsi de 57000 en 1999, à 71000 en 2000 et 81000 en 2001. Cette augmentation tranche avec l'évolution constatée depuis 1990. Après avoir connu une baisse importante entre 1993 et 1995 (de 60000 à 35 000), le nombre d'étrangers susceptibles d'entrer sur le marché du travail l'année même de leur arrivée en France à un autre titre que l'exercice d'une activité professionnelle s'était stabilisé au cours de la période 1996-1998: on en dénombrait environ 35000 en 1996, 37000 en 1997 et 39000 en 1998 (tableau 5). 
Tableau 5 : Flux annuel d'étrangers susceptibles d'entrer sur le marché du travail l'année même de leur arrivée en France à un autre titre que l'exercice d'une activité professionnelle au cours des années 1990-2001, selon le sexe et la nationalité regroupée

\begin{tabular}{|c|c|c|c|c|c|}
\hline \multirow{2}{*}{ Année } & \multicolumn{2}{|c|}{ EEE } & \multicolumn{2}{c|}{ Pays tiers } & \multirow{2}{*}{ Total } \\
\cline { 2 - 5 } & Hommes & Femmes & Hommes & Femmes & \\
\hline 1990 & 1067 & 2515 & 23915 & 30693 & 58190 \\
\hline 1991 & 1194 & 2520 & 27711 & 32980 & 64405 \\
\hline 1992 & 1660 & 3381 & 24591 & 29874 & 59506 \\
\hline 1993 & 1096 & 3169 & 24220 & 32131 & 60616 \\
\hline 1994 & 1216 & 2852 & 14949 & 22121 & 41138 \\
\hline 1995 & 822 & 2574 & 12515 & 18827 & 34738 \\
\hline $1996^{\star}$ & 1102 & 3741 & 12386 & 20689 & 37918 \\
\hline $1997^{\star}$ & 1561 & 4915 & 14988 & 24310 & 45774 \\
\hline $1998^{\star}$ & 1789 & 3967 & 18110 & 26384 & 50250 \\
\hline 1999 & 2628 & 5572 & 20026 & 29104 & 57330 \\
\hline 2000 & 3422 & 7688 & 25546 & 34673 & 71329 \\
\hline 2001 & 3378 & 7652 & 30707 & 39281 & 81018 \\
\hline
\end{tabular}

Source : d'après OMI, OFPRA, AGDREF, DPM (Lebon, 2001a, 2001b, 2003)

${ }^{*}$ ) Pour les étrangers originaires des pays tiers, y compris une partie des étrangers entrés en France comme « visiteurs » (cf. Léger, 2001).

Les étrangers originaires des pays tiers constituent l'essentiel (environ $85 \%$ ) des actifs potentiels au cours de la période 1999-2001. Parmi ces derniers, l'âge moyen des hommes est légèrement inférieur à celui des femmes (respectivement 29,7 ans et 30,5 ans). En revanche, parmi les actifs potentiels originaires de l'EEE, les femmes sont en moyenne nettement plus âgées que les hommes (respectivement 35 ans et 29 ans). Enfin, les hommes sont minoritaires : ils représentent, en moyenne, au cours de cette période, $31 \%$ des actifs potentiels originaires de l'EEE et $43 \%$ de ceux qui sont issus des pays tiers.

\section{Un actif potentiel sur deux entre sur le marché du travail}

En multipliant les effectifs d'actifs potentiels répartis par groupe d'âges, sexe et nationalité regroupée par les taux d'activité des étrangers ayant les mêmes caractéristiques démographiques, on estime le nombre d'« entrées indirectes » à 28000 en 1999, 35000 en 2000 et 41000 en 2001. Un actif potentiel sur deux entre donc, en moyenne, sur le marché du travail l'année même de son arrivée sur le territoire français (tableau 6).

Tableau 6 : Taux d'entrée (en \%) sur le marché du travail des actifs potentiels selon le sexe et la nationalité regroupée

\begin{tabular}{|c|c|c|c|c|c|}
\hline \multirow{2}{*}{ Année } & \multicolumn{2}{|c|}{ EEE } & \multicolumn{2}{|c|}{ Pays tiers } & \multirow{2}{*}{ Ensemble } \\
\hline & Hommes & Femmes & Hommes & Femmes & \\
\hline 1999 & 37 & 51 & 64 & 37 & 48 \\
\hline 2000 & 41 & 52 & 65 & 38 & 49 \\
\hline 2001 & 47 & 53 & 67 & 39 & 51 \\
\hline
\end{tabular}

En moyenne, au cours de la période 1999-2001, les ressortissants de l'EEE représentent seulement $14 \%$ de ce flux annuel d'entrées sur le marché du travail français. Parmi ces 
derniers, les hommes sont minoritaires ( $27 \%)$. Leur âge moyen ( 36,5 ans) est très proche de celui des femmes (37,2 ans). Chez les étrangers originaires des pays tiers ( $86 \%$ des entrées indirectes), les hommes sont en revanche plus nombreux que les femmes (respectivement $56 \%$ et $44 \%$ ), alors que parmi les actifs potentiels, c'est la situation inverse qui est observée. Ce renversement démographique est lié au fait que l'activité des hommes est beaucoup plus importante que celle des femmes (cf. supra, graphique 3), ce qui conduit à un taux d'entrée sur le marché du travail des actifs potentiels plus élevé pour les hommes. Pour les étrangers originaires des pays tiers, les hommes qui entrent sur le marché du travail ont en moyenne à peu près le même âge que les femmes (respectivement 32,3 ans et 32 ans).

Le nombre d'«entrées indirectes " estimé pour les années 2000 et 2001 est inédit, puisque ce niveau n'avait encore jamais été atteint depuis 1990 (tableau 7). De 1990 à 1993, on estimait à environ 30000 le nombre d'« entrées indirectes ». En 1994, la baisse du nombre d'actifs potentiels entraînait celle des «entrées indirectes » $(20000$ cette année-là). La baisse se poursuivait en 1995, année au cours de laquelle l'évaluation de ce flux atteignait sa valeur la plus basse depuis 1990 (17 000). Depuis 1996, le nombre estimé d'« entrées indirectes » n'a cessé d'augmenter. Le nombre croissant de titulaires de la carte « vie privée et familiale » parmi les actifs potentiels explique l'accélération de la croissance de ce flux depuis 1999.

Tableau 7 : Estimation de la série chronologique 1990-2001 des « entrées indirectes » selon la nationalité regroupée et le sexe

\begin{tabular}{|c|c|c|c|c|c|}
\hline \multirow{2}{*}{ Année } & \multicolumn{2}{|c|}{ EEE } & \multicolumn{2}{c|}{ Pays tiers } & \multirow{2}{*}{ Total } \\
\cline { 2 - 5 } & Hommes & Femmes & Hommes & Femmes & \\
\hline 1990 & 500 & 1500 & 16000 & 11000 & 29000 \\
\hline 1991 & 1000 & 1500 & 18500 & 12000 & 33000 \\
\hline 1992 & 1000 & 2000 & 16500 & 10500 & 30000 \\
\hline 1993 & 500 & 1500 & 16000 & 12000 & 30000 \\
\hline 1994 & 500 & 1500 & 9500 & 8500 & 20000 \\
\hline 1995 & 500 & 1500 & 8000 & 7000 & 17000 \\
\hline $1996^{\star}$ & 500 & 2000 & 8500 & 8000 & 19000 \\
\hline $1997^{\star}$ & 1000 & 3000 & 10500 & 9500 & 24000 \\
\hline $1998^{\star}$ & 1000 & 2000 & 12000 & 10000 & 25000 \\
\hline 1999 & 1000 & 3000 & 13000 & 11000 & 28000 \\
\hline 2000 & 1500 & 4000 & 16500 & 13000 & 35000 \\
\hline 2001 & 1500 & 4000 & 20500 & 15000 & 41000 \\
\hline
\end{tabular}

\section{Les « entrées différées »}

\section{Une estimation plus délicate, une précision plus limitée}

L'estimation des «entrées différées » se fait uniquement à partir des données relatives à la situation d'activité des étrangers, issues des enquêtes sur l'emploi de l'INSEE. Elle suppose de faire l'hypothèse que la courbe des taux d'activité par âge peut être assimilée, dans sa phase croissante, à la courbe des proportions cumulées de personnes qui sont entrées sur le marché du travail. Par exemple, à 30 ans, le taux d'activité des hommes originaires des pays non-membres de l'EEE est de $82 \%$. Selon cette hypothèse, on en déduit donc qu'à 30 ans, $82 \%$ des hommes originaires des pays tiers présents en 
France sont déjà entrés sur le marché du travail. Chaque taux d'activité par âge observé une année donnée correspond à une génération précise. Il traduit donc le comportement en matière d'activité professionnelle une année donnée des personnes nées au cours d'une même année. Or, en assimilant la série des taux d'activité par âge à la courbe des proportions cumulées de personnes qui sont entrées sur le marché du travail, on considère que les taux d'activité sont un reflet des comportements en matière d'activité des personnes issues d'une même génération. En faisant la première hypothèse, on en fait donc une seconde qui lui est liée : on considère que les taux d'activité par âge sont stables dans le temps. Cette hypothèse de stabilité dans le temps, non seulement de l'intensité de l'activité, mais aussi de son calendrier, ne s'observe généralement pas en pratique. Ainsi, au cours des années quatre-vingt par exemple, les taux d'activité aux âges les plus jeunes n'ont cessé de diminuer en raison de la progression généralisée de la durée de scolarisation. Toutefois, les dernières statistiques issues du ministère de l'éducation nationale montrent que la durée des études tend à se stabiliser depuis quelques années : ces dernières années, les taux de scolarisation sont restés inchangés avant 18 ans et au-delà de 21 ans (PouletCoulibando, Zamora, 2000). Le calendrier de sortie du système éducatif semblant se stabiliser, celui des entrées sur le marché du travail ne devrait plus beaucoup évoluer.

Comme la quasi-totalité des hommes est active, on peut considérer que l'assimilation des taux d'activité aux proportions cumulées d'entrées sur le marché de l'emploi est, pour la population masculine, une hypothèse légitime. On ne peut pas être aussi affirmatif pour les femmes. En effet, leur trajectoire professionnelle est caractérisée par une moindre continuité que celle des hommes. Quand les hommes quittent le marché de l'emploi, c'est le plus souvent pour prendre leur retraite. La cessation d'activité est le plus souvent définitive, tandis que pour un certain nombre de femmes, la sortie du marché de l'emploi peut être temporaire, pour des raisons familiales notamment. La stabilité du taux d'activité à partir de 25 ans, à un niveau moindre que celui observé pour les hommes, est en partie la conséquence de mouvements d'entrées/sorties de la catégorie des actifs. Il devient alors plus difficile d'assimiler les taux d'activité à un cumul d'entrées sur le marché du travail. Nous l'avons pourtant fait en projetant la courbe, au-delà de 25 ans environ, sur la base de sa pente entre quinze et vingt-cinq ans, et en faisant tendre celle-ci vers le taux d'activité le plus élevé observé.

Les résultats auxquels parviennent cette méthode conduisent à estimer l'intensité d'entrée sur le marché du travail à plus de $95 \%$ pour les hommes quelle que soit leur nationalité, à $90 \%$ pour les femmes originaires des pays membres de l'EEE et à près de $60 \%$ pour celles originaires des pays tiers. Le calendrier d'entrée sur le marché du travail est, d'après cette méthode d'estimation, assez proche quels que soient le sexe et le groupe de nationalité : la moitié des entrées sur le marché de l'emploi se fait avant 21 ans pour les hommes, 21,5 ans pour les femmes originaires des pays tiers et 22 ans pour celles originaires des pays membres de l'EEE (graphique 4). 
Graphique 4 : Estimation des fonctions d'entrée sur le marché du travail selon le sexe et la nationalité regroupée

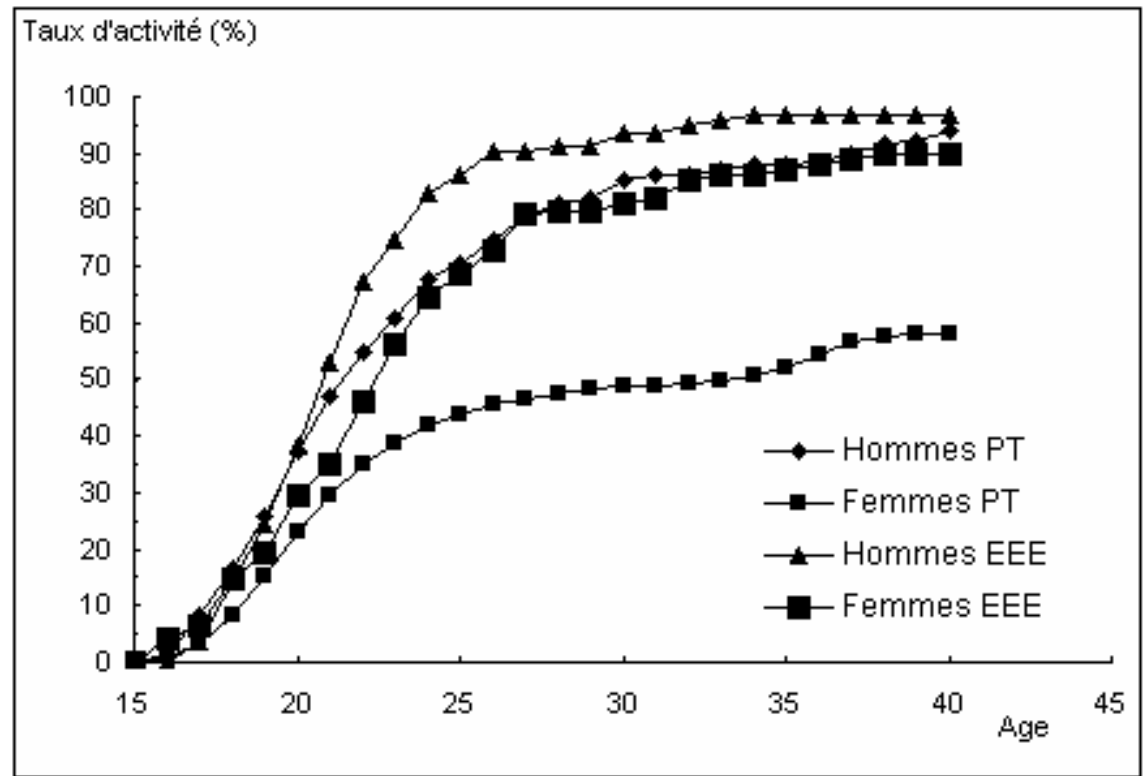

Une fois ces hypothèses admises, on parvient à estimer le nombre d'étrangers, présents en début d'année en France, susceptibles d'entrer pour la première fois sur le marché du travail, en multipliant les proportions cumulées de personnes encore jamais entrées sur le marché du travail par les effectifs d'étrangers présents en France. On applique alors aux effectifs d'actifs potentiels ainsi obtenus et répartis selon la nationalité regroupée, le sexe et l'âge, les probabilités correspondantes de devenir actif. Celles-ci correspondent au complément à l'unité du rapport entre le taux d'inactivité à l'âge $\mathrm{x}$ et le taux d'inactivité à l'âge x-1 (probabilité de rester inactif dans l'année quand on est âgé de $\mathrm{x}$ ans).

Les hypothèses sous-jacentes à cette estimation sont les mêmes que celles sur lesquelles l'INSEE fonde son calcul de l'âge moyen d'entrée sur le marché du travail (Brondel, Guillemot, Marioni, 1996). Mais cette estimation suppose aussi que la population étrangère soit une population fermée, c'est-à-dire que les sorties de cette population soient négligeables. Cette hypothèse, légitime quand on travaille sur l'ensemble de la population française, est plus problématique quand on considère seulement la population étrangère. En effet, elle suppose que les départs de la catégorie "étranger ", soit par décès, soit par sortie du territoire, soit par acquisition de la nationalité française, sont sans conséquence sur le stock présent en début d'année. Présentée autrement, cela revient à intégrer dans le flux d'«entrées différées» les étrangers présents en France en début d'année, quel que soit leur devenir (décès, départ, naturalisation) au cours de l'année. Cette hypothèse d'une population fermée, qui simplifie la démarche d'estimation, a été testée et il s'est avéré que la prise en compte de ces différentes "sorties» de la catégorie étranger avaient peu d'incidence sur l'ordre de grandeur du nombre d'« entrées différées » (Léger, 2003). 
Tableau 8 : Estimation de la série chronologique 1990-2001 des « entrées différées » selon la nationalité

\begin{tabular}{|c|c|c|c|c|c|}
\hline \multirow{2}{*}{ Année } & \multicolumn{2}{|c|}{ EEE } & \multicolumn{2}{c|}{ Pays tiers } & \multirow{2}{*}{ Total } \\
\cline { 2 - 5 } & Hommes & Femmes & Hommes & Femmes & \\
\hline 1990 & 8000 & 7000 & 15000 & 10000 & 40000 \\
\hline 1991 & 8000 & 6000 & 15000 & 9000 & 38000 \\
\hline 1992 & 7000 & 6000 & 15000 & 10000 & 38000 \\
\hline 1993 & 7000 & 5000 & 17000 & 11000 & 40000 \\
\hline 1994 & 5000 & 4000 & 18000 & 12000 & 39000 \\
\hline 1995 & 4000 & 3000 & 16000 & 11000 & 34000 \\
\hline 1996 & 4000 & 3000 & 14000 & 11000 & 32000 \\
\hline 1997 & 4000 & 3000 & 14000 & 11000 & 32000 \\
\hline 1998 & 4000 & 3000 & 14000 & 11000 & 32000 \\
\hline 1999 & 4000 & 4000 & 13000 & 10000 & 31000 \\
\hline 2000 & 4000 & 4000 & 13000 & 10000 & 31000 \\
\hline 2001 & 4000 & 4000 & 13000 & 10000 & 31000 \\
\hline
\end{tabular}

Remarque : Estimation à partir de données quinquennales pour les années 1990-1998 et de données par âge pour la période 1999-2001.

\section{Les « entrées différées » sont surtout le fait de jeunes actifs}

Le nombre annuel d'« entrées différées » ainsi estimé pour les années 1999-2001 s'élève à près de 31000 , dont 8000 ressortissants des pays membres de l'EEE (27 \%). Parmi ces derniers, les hommes sont plus nombreux que les femmes (respectivement $54 \%$ et $46 \%$ ). À peu de choses près, on observe le même phénomène chez les étrangers originaires des pays tiers : les hommes et les femmes représentent respectivement $58 \%$ et $42 \%$ des « entrées différées » issues de ce groupe de nationalités. Enfin, les « entrées différées ", telles que nous les avons estimées pour cette dernière période, sont le fait de jeunes actifs ( $65 \%$ sont âgés de moins de 25 ans) $)^{5}$.

Jamais l'effectif de ce flux n'a été aussi faible depuis que cette estimation est réalisée : on a en effet estimé à environ 39000 le nombre d'« entrées différées » au cours des années 1990-1992, 38000 pendant la période 1993-1995 et 32000 au cours des années 1996-1998 (tableau 8).

\section{Les bénéficiaires du réexamen et de son prolongement}

En 1997, à l'initiative des Pouvoirs publics, a commencé l'opération de régularisation sur critères de la situation administrative d'un certain nombre d'étrangers. La plupart de ces bénéficiaires du réexamen de 1997 sont actifs. En effet, l'analyse d'un échantillon de 11000 cartes délivrées durant le second semestre 1997 a révélé que 10300 d'entre elles environ (soit près de $94 \%$ ) étaient des premiers titres "salariés". Or, ces bénéficiaires n'entrent pas sous la rubrique "travailleurs permanents " dans les statistiques de l'OMI (Lebon, 2000). Les renseignements pour l'année 1999 ont confirmé cette proportion (Lebon, 2001a). Si l'entrée sur le marché du travail français de la plupart de ces étrangers est antérieure à l'année au cours de laquelle leur situation de séjour est régularisée, ils apparaissent néanmoins pour la première fois, sur le plan 
légal et statistique, parmi les actifs étrangers présents en France. C'est pourquoi ils ont été inclus dans le champ de l'estimation du flux annuel d'entrées sur le marché du travail français.

À partir de 1999, l'opération de régularisation proprement dite étant achevée, le nombre de bénéficiaires a chuté : il est de 3322 en 1999, 163 en 2000 et 65 en 2001, tandis qu'il était de 18910 en 1997 et de 45773 en 1998. Toutefois, cette diminution est atténuée par l'apparition statistique de ceux des étrangers qui ont reçu la carte «vie privée et familiale ", soit parce qu'ils résident habituellement en France depuis plus de 10 ans ou plus de 15 ans en qualité d'étudiant, soit en raison de l'existence de « liens personnels et familiaux ». Avec l'apport de ce type de titulaires de la carte "vie privée et familiale", le flux annuel de bénéficiaires du réexamen de 1997 et de son prolongement au cours de la période 1999-2001 est supérieur à 8000 entrées. En considérant que $90 \%$ d'entre eux sont des actifs, on peut évaluer leur effectif à 8000 en 1999 et à 7000 en 2000 et 2001 (tableau 9).

Tableau 9 : Estimation du nombre annuel d'actifs originaires des pays tiers qui ont bénéficié du réexamen de 1997 et de son prolongement

\begin{tabular}{|c|c|c|c|}
\hline \multirow{2}{*}{ Année } & \multicolumn{2}{|c|}{ Pays tiers } & \multirow{2}{*}{ Total } \\
\cline { 2 - 3 } & Hommes & Femmes & \\
\hline 1997 & 8000 & 10000 & 18000 \\
\hline 1998 & 22000 & 19000 & 41000 \\
\hline 1999 & 5000 & 3000 & 8000 \\
\hline 2000 & 4500 & 3000 & 7500 \\
\hline 2001 & 4000 & 3500 & 7500 \\
\hline
\end{tabular}

Remarque : À partir de 1999, les effectifs correspondent aux bénéficiaires du réexamen de 1997 ainsi qu'aux étrangers qui ont obtenu une carte de séjour « vie privée et familiale » pour un motif prolongeant l'opération de réexamen.

Depuis 1997, la répartition selon le sexe des bénéficiaires du réexamen de 1997 et de son prolongement, qui sont entrés, d'un point de vue statistique, sur le marché du travail français, a connu des variations importantes. Alors que les hommes sont minoritaires en 1997 (44\%), ils deviennent nettement majoritaires l'année suivante (54\%), avant de représenter plus de $60 \%$ de ce flux au cours des années 1999 et 2000. En 2001, leur proportion est redescendue à $54 \%$. La structure par âge a elle aussi connu quelques variations. Ainsi, l'âge moyen des étrangers qui ont bénéficié du réexamen de 1997 en tant qu'actifs est de 33,4 ans en 2001, soit un an de moins que celui calculé pour l'année 1999 (34,4 ans). En revanche, il se dégage une constante : quelle que soit l'année considérée, les femmes sont en moyenne plus jeunes que les hommes. Au cours des années 1999-2001, elles sont $61 \%$ à être âgées de moins de 35 ans, contre $54 \%$ des hommes. Cette différence se traduit par un écart d'âge moyen d'une année : il est en effet de 33,2 ans pour les femmes et 34,3 ans pour les hommes au cours des années 1999-2001. 


\section{L'évolution du flux annuel global de 1990 à 2001}

d'étrangers qui ont bénéficié du réexamen de 1997 et de son prolongement en tant qu'actifs, conduit à un flux annuel d'entrées d'étrangers sur le marché du travail de 102000 en 2001. C'est plus qu'en 2000 (93 500 entrées estimées) et 1999 (86 000 entrées estimées). En plus de cette évolution quantitative, la composition de ces entrées a varié d'une année à l'autre au cours de la période 1999-2001. Ainsi :

- les « entrées différées » représentent $36 \%$ des entrées annuelles d'étrangers sur le marché de l'emploi en 1999, $33 \%$ en 2000 et $30 \%$ en 2001 ;

- les « entrées indirectes » représentent $32 \%$ de ce flux annuel de nouveaux actifs en 1999, $38 \%$ en 2000 et $41 \%$ en 2001 ;

En revanche, la part des « entrées directes » et des bénéficiaires du réexamen de 1997 et de son prolongement est restée stable d'une année à l'autre. Ainsi :

- - les « entrées directes » représentent $22 \%$ des entrées annuelles d'étrangers sur le marché de l'emploi en 1999, $21 \%$ en 2000 et $22 \%$ en 2001 ;

- - les bénéficiaires du réexamen de 1997 et de son prolongement constituent respectivement $9 \%, 8 \%$ et $7 \%$ des entrées annuelles d'étrangers sur le marché de l'emploi en 1999, 2000 et 2001.

resstissants de l'EEE représentent plus du quart de ce flux (27\% en 2001). Parm eux, on compte plus d'hommes que de femmes (respectivement $55 \%$ et $45 \%$ ). Chez les étrangers originaires des pays tiers, la prééminence des hommes est encore plus marquée ( $58 \%$ contre $42 \%$ ). Ces résultats sont comparables à ceux obtenus depuis 1990 . Enfin, sur le plan de l'âge, les ressortissants de l'EEE sont un peu plus âgés, en moyenne, que les étrangers originaires des pays tiers (respectivement 32 ans et 30 ans).

Les résultats obtenus pour les années 1999-2001 s'inscrivent dans le cadre de l'augmentation tendancielle du flux annuel d'entrées d'étrangers sur le marché du travail observée depuis 1997, après une phase de forte décroissance (tableau 10). La série statistique 1990-2001 peut en effet être scindée en deux grandes phases. La première couvre les années 1990-1996. Elle est caractérisée par une baisse régulière du nombre annuel d'entrées d'étrangers sur le marché du travail français : si l'on excepte l'année 1992, le flux annuel de nouveaux actifs étrangers est passé en sept ans de 100000 à moins de 70000 . À partir de 1997, sous l'effet du réexamen décidé cette année-là, les entrées d'actifs étrangers ont de nouveau augmenté pour atteindre les 116000 en 1998, parmi lesquelles plus de 40000 sont le fait de régularisés devenus actifs. Depuis, le nombre d'étrangers qui ont profité de cette mesure n'a cessé de diminuer. En 1999 et après, avec la prise en compte des premiers titulaires de la carte « vie privée et familiale », la tendance à la hausse observée depuis 1997 s'est maintenue. En effet, si l'on exclue l'apport des bénéficiaires du réexamen, au sens où on l'entend ici, le flux annuel de nouveaux actifs étrangers est passé de 66500 entrées en 1996 à 72000 en 1997, 75000 en 1998, 78000 en 1999, 86000 en 2000 et 94500 en 2001 (tableau 11). 
Tableau 10 : Bilan de l'estimation des entrées annuelles d'étrangers sur le marché du travail français de 1990 à 2001, selon la nationalité regroupée et le sexe

\begin{tabular}{|c|c|c|c|c|r|}
\hline \multirow{2}{*}{ Année } & \multicolumn{2}{|c|}{ EEE } & \multicolumn{2}{c|}{ Pays tiers } & \multirow{2}{*}{ Total } \\
\cline { 2 - 5 } & Hommes & Femmes & Hommes & Femmes & \\
\hline 1990 & 14500 & 12500 & 45000 & 26000 & 98000 \\
\hline 1991 & 15000 & 11500 & 50000 & 26500 & 103000 \\
\hline 1992 & 24000 & 18000 & 51000 & 27000 & 120000 \\
\hline 1993 & 17500 & 13000 & 41000 & 27000 & 98500 \\
\hline 1994 & 13500 & 11000 & 33500 & 23000 & 81000 \\
\hline 1995 & 11000 & 8500 & 29000 & 21500 & 70000 \\
\hline $1996^{*}$ & 11000 & 9000 & 26000 & 20500 & 66500 \\
\hline $1997^{\star}$ & 11500 & 10500 & 36000 & 32000 & 90000 \\
\hline $1998^{*}$ & 13000 & 10000 & 51500 & 41500 & 116000 \\
\hline 1999 & 13000 & 11500 & 35500 & 26000 & 86000 \\
\hline 2000 & 14500 & 12500 & 38500 & 28000 & 93500 \\
\hline 2001 & 14500 & 12500 & 44000 & 31000 & 102000 \\
\hline
\end{tabular}

Tableau 11 : Bilan de l'estimation des entrées annuelles d'étrangers sur le marché du travail français de 1990 à 2001, selon le type d'entrée

\begin{tabular}{|c|c|c|c|c|c|}
\hline \multirow[b]{2}{*}{ Année } & \multicolumn{4}{|c|}{ Type d'entrée } & \multirow[b]{2}{*}{ Total } \\
\hline & "Directes" & "Indirectes» & « Différées » & $\begin{array}{l}\text { Réexamen } \\
\text { de } 1997^{\star}\end{array}$ & \\
\hline 1990 & 29000 & 29000 & 40000 & & 98000 \\
\hline 1991 & 32000 & 33000 & 38000 & & 103000 \\
\hline 1992 & 52000 & 30000 & 38000 & & 120000 \\
\hline 1993 & 28500 & 30000 & 40000 & & 98500 \\
\hline 1994 & 22000 & 20000 & 39000 & & 81000 \\
\hline 1995 & 17000 & 19000 & 34000 & & 70000 \\
\hline 1996 & 15500 & 19000 & 32000 & & 66500 \\
\hline 1997 & 16000 & 24000 & 32000 & 18000 & 90000 \\
\hline 1998 & 18000 & 25000 & 32000 & 41000 & 116000 \\
\hline 1999 & 19000 & 28000 & 31000 & 8000 & 86000 \\
\hline 2000 & 20000 & 35000 & 31000 & 7500 & 93500 \\
\hline 2001 & 22500 & 41000 & 31000 & 7500 & 102000 \\
\hline
\end{tabular}

Depuis 2000, les «entrées indirectes» constituent le principal flux d'entrées d'actifs étrangers sur le marché du travail français. Au cours des années antérieures, sauf en 1992 et en 1998, ce sont les "entrées différées" qui ont le plus participé au renouvellement de la population active étrangère. Au cours des années 1994-1996, elles ont même représenté la moitié du flux annuel de nouveaux actifs étrangers. En 1992, l'élargissement de la libre circulation aux ressortissants espagnols et portugais, ainsi que la régularisation de demandeurs d'asile déboutés, expliquent l'augmentation du nombre d'«entrées directes " qui, cette année-là, ont constitué le principal flux d'entrées d'actifs étrangers sur le marché du travail français (43\%). En 1998, la vague de régularisation consécutive au réexamen de 1997 a fait des bénéficiaires de cette procédure le principal flux d'entrées d'étrangers sur le marché de l'emploi français (35\%).

36 Le poids relatif de chaque type d'entrées sur le marché du travail varie d'un groupe de nationalités à l'autre. Depuis 1992, les «entrées directes » représentent au moins la 
moitié (jusqu'à 63 \% en 1992) des entrées annuelles sur le marché du travail français des étrangers originaires des pays membres de l'EEE. En 1990 et 1991, ce sont les « entrées différées » qui ont représenté la majorité du flux annuel de nouveaux actifs originaires de l'EEE. Enfin, on peut noter que le poids des « entrées indirectes » a triplé en douze ans, passant de $7 \%$ de l'ensemble des entrées d'étrangers originaires de l'EEE sur le marché du travail français en 1990 à $21 \%$ en 2001 (graphique 5).

Graphique 5 : Répartition (en \%) des entrées d'étrangers originaires des pays membres de l'EEE sur le marché du travail français selon le type d'entrée de 1990 à 2001

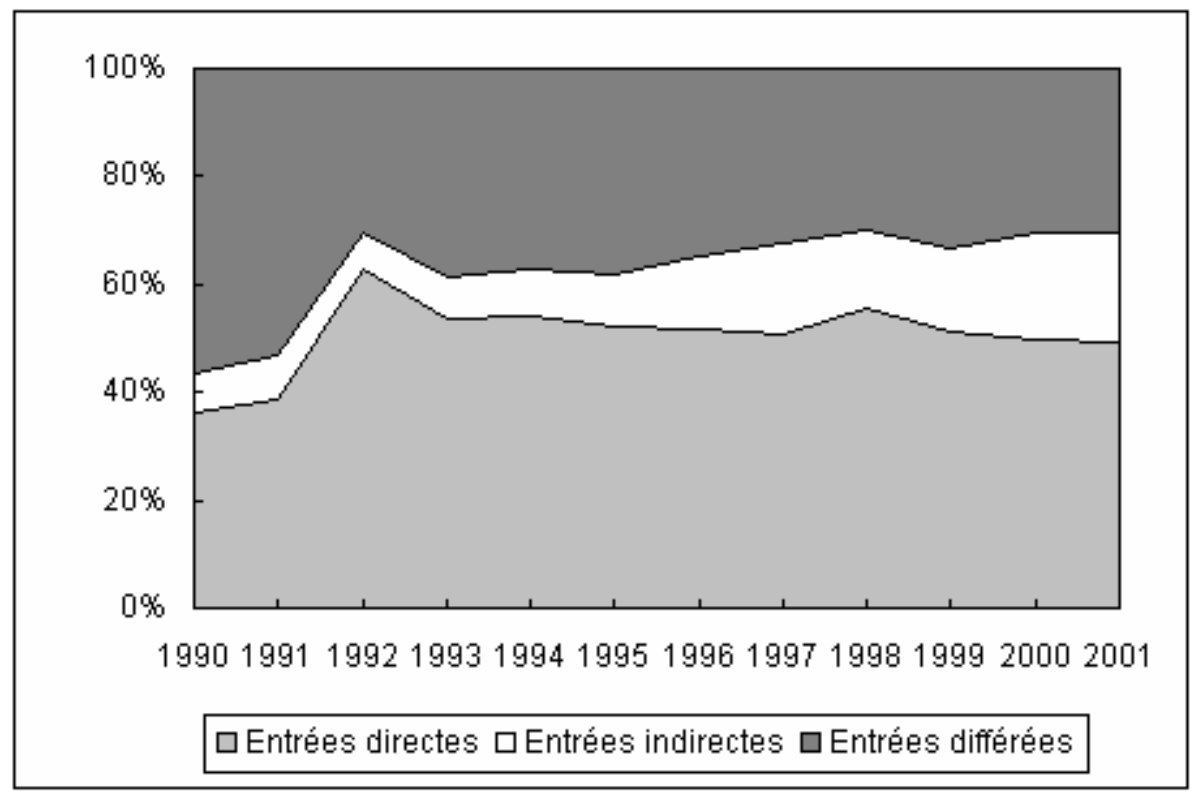

Pour les étrangers originaires des pays tiers, les « entrées indirectes » et les « entrées différées » ont été, à tour de rôle, les principaux flux d'entrées sur le marché du travail français depuis 1990. Si les « entrées indirectes » ont constitué le principal flux au cours des années 1990-1992, la répartition selon le type d'entrée sur le marché du travail était toutefois relativement équilibrée. Ainsi, en 1992, on comptait $33 \%$ d'« entrées directes ", $35 \%$ d'« entrées indirectes » et $32 \%$ d'« entrées différées ». La baisse spectaculaire du nombre d'« entrées directes » d'étrangers originaires des pays tiers en 1993 a rompu cet équilibre. Cette année-là, les étrangers qui sont entrés en France afin d'exercer une activité professionnelle ne représentaient plus que $17 \%$ du flux d'entrées d'étrangers originaires des pays tiers sur le marché du travail français. Depuis 1994, les «entrées directes» représentent moins de $15 \%$ du flux annuel de nouveaux actifs originaires des pays tiers. La baisse observée du nombre d'«entrées indirectes» explique que les «entrées différées » soient devenues, entre 1993 et 1997, le principal mode d'accès au marché du travail français. Les bénéficiaires du réexamen de 1997 vont atténuer l'importance relative de ce flux, notamment en 1998, année au cours de laquelle ils représentent près d'un nouvel actif sur deux (44\%). Sous l'impulsion des titulaires de la carte de séjour "vie privée et familiale ", les « entrées indirectes » sont redevenues, depuis 1999, le principal flux d'entrées d'actifs étrangers originaires des pays tiers sur le marché du travail français (graphique 6). 
Graphique 6 : Répartition (en \%) des entrées d'étrangers originaires des pays tiers sur le marché du travail français selon le type d'entrée de 1990 à 2001

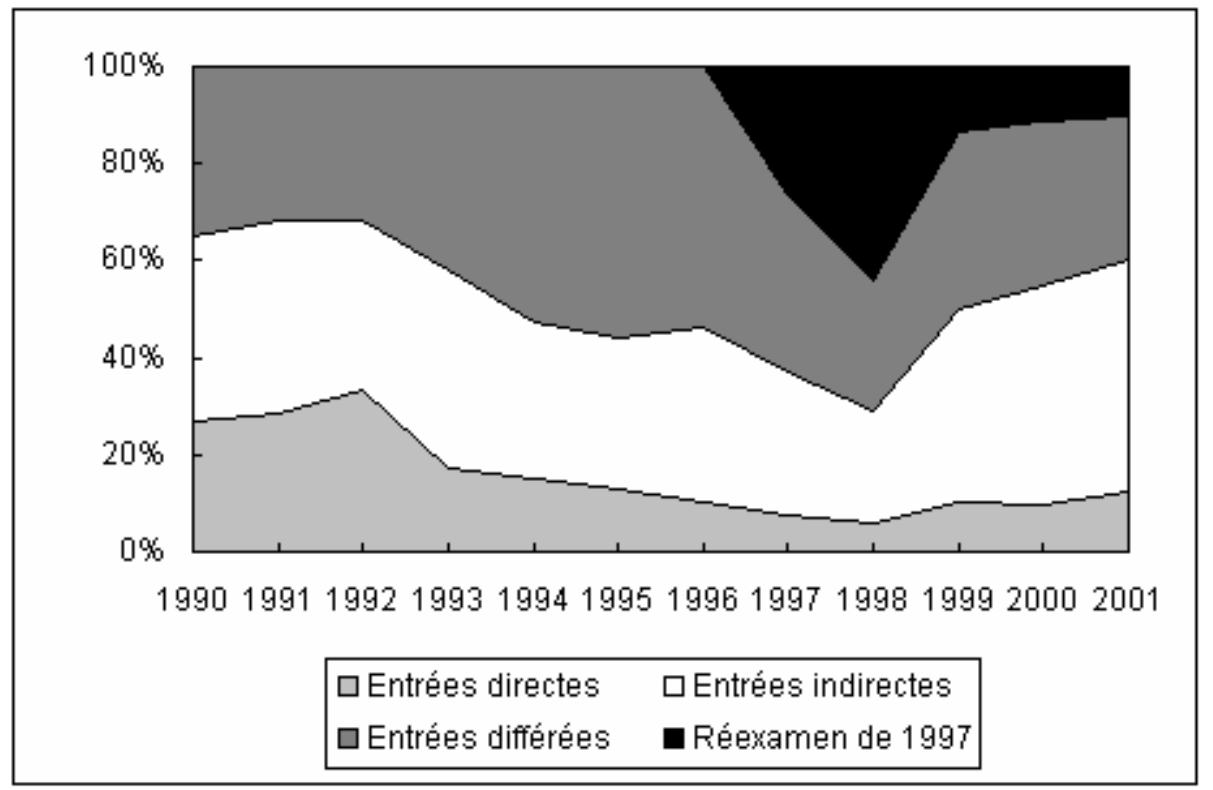

\section{Conclusion : plus d'un nouvel actif sur dix est étranger}

Au terme de cette étude, on peut s'interroger sur la place que tiennent les étrangers dans le flux global d'entrées sur le marché du travail français. Afin de proposer une mesure de l'ordre de grandeur du poids des étrangers parmi l'ensemble des nouveaux actifs une année donnée, il est nécessaire, au préalable, d'estimer ce flux composé, d'une part, d'entrées "internes» (ensemble des entrées de personnes présentes en France le 1er janvier de l'année, quelle que soit leur nationalité, y compris les « entrées différées ») et, d'autre part, d'entrées "externes» (elles rassemblent les "entrées directes" et "indirectes» d'étrangers, ainsi que les étrangers qui ont bénéficié du réexamen de 1997 et de son prolongement en tant qu'actifs). Les entrées "internes » ont été estimées à partir des données des enquêtes sur l'emploi de l'INSEE en utilisant la même méthode que celle, utilisée pour l'évaluation des « entrées différées ». De cette manière, on évalue à environ 670000 le nombre annuel d'entrées «internes» sur le marché de l'emploi pour les années 1999-2001. Le flux annuel global d'entrées sur le marché du travail français est donc estimé, avec la prise en compte des entrées « externes », à 725000 en 1999, 732500 en 2000 et 741000 en 2001. Sur cette base méthodologique, la proportion d'actifs étrangers est évaluée à $12 \%$ en $1999,13 \%$ en 2000 et $14 \%$ en 2001. Ces résultats sont comparables à ceux obtenus pour les années antérieures : cette proportion a en effet été estimée à $13 \%$ en 1990 et $1991,16 \%$ en 1992, 13 \% en 1993, 11 \% en 1994, 9 \% en 1995 et 1996, 12 \% en 1997 et $15 \%$ en 1998.

Depuis une dizaine d'années, les étrangers représentent environ $6 \%$ des actifs présents sur le marché de l'emploi français, soit deux fois moins que la part estimée des étrangers dans le flux annuel global d'entrées sur le marché du travail français. Doit-on voir dans cet écart les limites de notre mode d'estimation? Le fait que la part des étrangers dans le flux d'entrées d'actifs sur le marché de l'emploi français soit plus importante que la proportion d'étrangers dans le stock d'actifs ne constitue pas un 
résultat incohérent. Comme les flux globaux de la population active sont stables, cela suppose simplement que le nombre de sorties de la catégorie "actifs étrangers " soit équivalent aux nombres d'entrées d'actifs étrangers sur le marché du travail français. Mais cela ne suppose pas, pour autant, que la part des étrangers dans le flux de sorties d'actifs soit équivalente à la part de ces derniers dans le flux d'entrées sur le marché du travail.

Pour les nationaux, les sorties du marché du travail résultent de trois causes d'ampleur variable : les cessations d'activité (par retraite ou par démission), l'expatriation et les décès. Les étrangers, en revanche, sont les seuls à connaître un motif supplémentaire dont l'importance a augmenté au cours des dernières années: l'acquisition de la nationalité française. Dans ce cas, en effet, s'ils demeurent bien actifs, ils ne sont plus dénombrés en tant qu'actifs étrangers. Dès lors, pour que le nombre d'actifs étrangers reste constant, ces sorties particulières de la catégorie " actifs étrangers » doivent être compensées. C'est la raison pour laquelle les entrées d'actifs étrangers doivent nécessairement être, chaque année, plus nombreuses que le total des départs en retraite, sorties du territoire, démissions et décès d'actifs étrangers. De ce fait, la proportion d'étrangers dans le flux d'entrées sur le marché de l'emploi est nécessairement plus élevée que la part de ces derniers dans le flux de sorties de la catégorie "actifs", dont on peut penser qu'elle est au moins égale à la proportion d'étrangers parmi les actifs français, c'est-à-dire $6 \%$.

41 Cette confrontation rappelle toutefois que, compte tenu des hypothèses mises en œuvre dans le cadre de cette méthode d'estimation du flux annuel d'entrées d'étrangers sur le marché du travail, les résultats auxquels nous parvenons ne peuvent être considérés comme des résultats précis. Néanmoins, malgré leur caractère approximatif, ils permettent d'établir un ordre de grandeur vraisemblable du flux annuel d'entrées d'actifs étrangers sur le marché national de l'emploi au cours des années 1990-2001. En tout cas, il apparaît clairement que, sur cette période, celui-ci ne saurait être réduit aux seuls étrangers qui entrent en France afin d'exercer une activité professionnelle, qui représentent, selon notre estimation, environ un nouvel actif étranger sur quatre.

\section{BIBLIOGRAPHIE}

BRONDEL D., GUILLEMOT D., MARIONI P. (1996) « La population active : facteurs d'évolution et perspectives », Données sociales 1996, INSEE, pp. 110-115.

LEBON André (1993) Immigration et présence étrangère en France. Le bilan d'une année 1992/1993, la Documentation française.

LEBON André (2000) Migrations et nationalité en France en 1998, la Documentation française.

LEBON André (2001 (a)) Immigration et présence étrangère en France en 1999. Premiers enseignements du recensement, la Documentation française. 
LEBON André (2001 (b)) Migrations et nationalité en France en 2000, Rapport au SOPEMI (OCDE), Ministère de l'emploi et de la solidarité.

LEBON André (2003) Migrations et nationalité en France en 2001, la Documentation française.

LÉGER Jean-François (2001) « Le renouvellement de la population active étrangère en France de 1990 à 1998 ", Revue européenne des migrations internationales, vol. 17, n² 2, pp. 101-121.

LÉGER Jean-François (2003) Les entrées d'étrangers sur le marché de l'emploi français de 1999 à 2001, rapport à l'attention de la Direction de la population et des migrations, Ministère des affaires sociales, du travail et de la solidarité.

POULET-COULIBANDO P. et ZAMORA (2000) «Insertion des jeunes : sensible amélioration, surtout chez les diplômés », INSEE Première, $\mathrm{n}^{\circ} 741$.

\section{ANNEXES}

\section{ANNEXE 1 : Précisions méthodologiques concernant l'estimation des « entrées directes » au cours des années 1990-1996}

L'estimation rétrospective des « entrées directes » issues de l'EEE pour les années 1990-1996 se fonde sur la comparaison des écarts observés entre les statistiques de l'OMI et celles issues de l'AGDREF.

Dans un premier temps, nous avons calculé la différence absolue observée, pour les années 1997-2001, entre l'effectif donné par les statistiques issues de l'AGDREF et les effectifs de référence estimés par la DPM.

Puis, nous avons déterminé la différence relative entre les variations absolues observées d'une année à l'autre. Ce rapport est égal à 0,93 entre les années 2000 et 1999, et 1999 et 1998 ; il est égal à 0,65 entre les années 1998 et 1997. Nous avons considéré que ce dernier rapport demeurait stable à partir de 1997 jusqu'en 1990.

En appliquant ce cœfficient à l'écart absolu observé entre le flux « AGDREF » et le flux « DPM » pour l'année 1997, on estime la différence absolue qu'on aurait observée en 1996 entre ces deux sources d'information. On renouvelle la même opération pour les années suivantes. On obtient alors toute une série d'écarts entre les effectifs de référence de la DPM et les données AGDREF si elles étaient connues pour les années antérieures à 1997 (graphique A. 1). Ce principe d'estimation conduit à minimiser l'écart, au fur et à mesure que l'on « remonte » dans le temps, entre les estimations fournies par la DPM et les données AGDREF antérieures à 1997.

Enfin, on ajoute aux estimations du flux annuel d'« entrées directes » issues de l'EEE proposées depuis 1990 par la DPM les écarts ainsi estimés. On obtient alors une estimation de ce flux d'actifs pour ce groupe de nationalités pour les années 1990-1996. 
Graphique A.1 : Estimation des écarts entre les estimations des « entrées directes » issues de l'EEE produites par la DPM et les données AGDREF antérieures à 1997

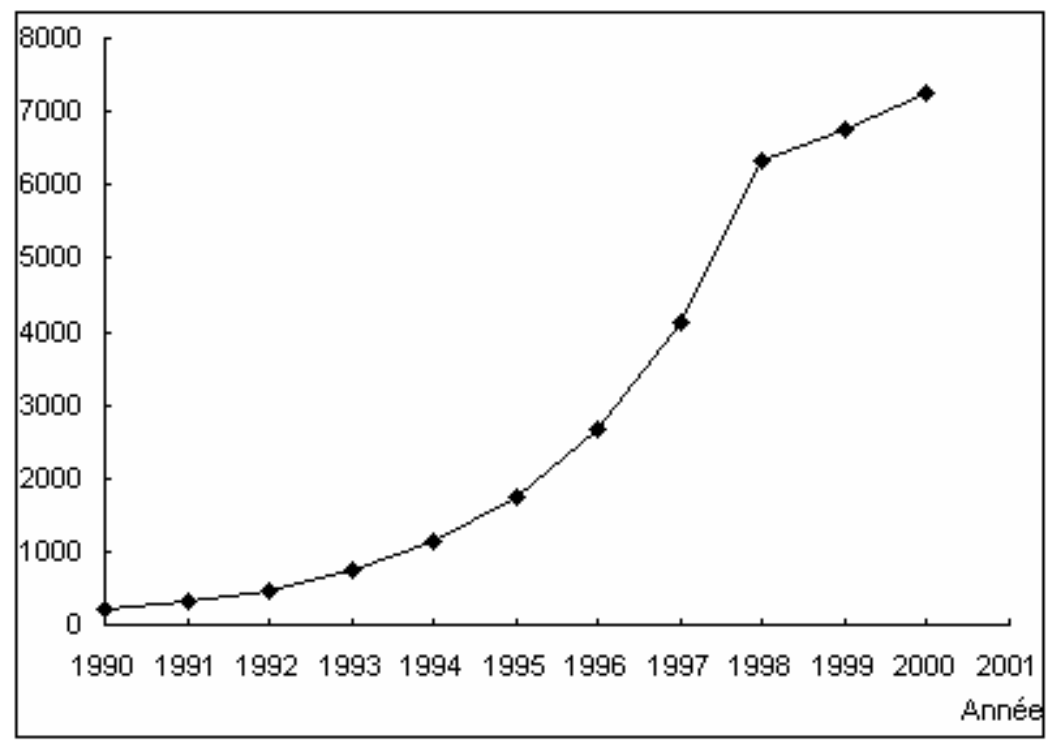

\section{ANNEXE 2 : Effets sur l'estimation des « entrées différées " de l'utilisation des taux d'activité par âge à la place de taux d'activité par groupe d'âges quinquennal}

Dans nos précédentes estimations du nombre d'étrangers qui entrent chaque année sur le marché du travail (Léger, 2001), les « entrées différées » ont été estimées à partir des taux d'activité par groupe d'âges quinquennal. Ces données nous étaient alors fournies par l'INSEE. Mais depuis 1999, l'INSEE commercialise le fichier de l'enquête Emploi, ce qui permet de travailler sur des données plus fines. De ce fait, pour les années 1999-2001, nous avons estimé les « entrées différées » à partir de la série des taux d'activité par âge. Bien que le principe d'estimation soit le même, ce changement a des conséquences sur les résultats de l'estimation. Ainsi, la méthode fondée sur l'utilisation de données par âge conduit à une estimation de l'ordre de 30500 " entrées différées ", tandis que celle basée sur des données regroupées par âge quinquennal parvient à 27500 « entrées différées ». La différence (3 000 en faveur de l'estimation à partir des données par âge) est particulièrement nette pour les ressortissants de l'EEE (+ 2 250). Enfin, sur le plan de la structure par âge, l'utilisation de données quinquennales conduit à une structure par âge plus « jeune » $(50 \%$ des « entrées différées » avant 20 ans, $75 \%$ avant 25 ans, tandis qu'à partir des données par âge on obtient respectivement des pourcentages voisins de $30 \%$ et $60 \%$ ).

L'utilisation de données par âge conduit à des taux d'activité moins importants aux jeunes âges, ce qui explique le « vieillissement » des « entrées différées » quand on utilise ces statistiques. Mais dans le même temps, les actifs potentiels estimés à partir de ces données sont plus nombreux que ceux obtenus à partir des données par groupe d'âges quinquennal. Même si les probabilités estimées d'accès au marché de l'emploi sont moins élevées à ces mêmes âges, leur effet sur le volume des « entrées différées » 
auxquelles cette méthode parvient est moindre que celui du nombre plus important d'actifs potentiels. Par conséquent, l'utilisation de données par âge conduit à une estimation de l'effectif des « entrées différées » un peu supérieure (mais aussi plus juste) que celle à laquelle parvient le recours à des données par groupe d'âges quinquennal. Toutefois, compte tenu des hypothèses sur lesquelles se fonde ce calcul, on peut admettre que le changement de type de statistiques est sans conséquence véritablement notable sur l'ordre de grandeur de l'estimation de ce flux.

\section{NOTES}

1. Je tiens à remercier Chantal Daufresne (OMI) et Guy Lucas (ministère de l'intérieur) pour l'ensemble des données qu'ils m'ont l'un et l'autre transmis sous forme de fichier informatique, ainsi que pour la disponibilité dont ils ont fait preuve chaque fois que je les ai sollicités. Par ailleurs, Catherine Borrel (INSEE) m'a permis d'apporter d'utiles précisions méthodologiques. Enfin, par sa disponibilité et sa connaissance des caractéristiques de l'immigration et de la présence étrangère en France, André Lebon (DPM) a contribué de manière décisive à l'élaboration de ce travail. Ce texte a également bénéficié de ses remarques, ainsi que de celles d'Yves Charbit (Université Paris V). Les résultats présentés dans cet article ne sauraient cependant engager leur responsabilité.

2. Dans cet article, le libellé « EEE » correspond à la fois aux pays membres de l'UE à 12 (pour les années 1990-1993) et à l'ensemble constitué des pays membres de l'UE à 12 et de l'Autriche, la Finlande, l'Islande, la Norvège et la Suède (à partir du 1er janvier 1994).

3. L'AGDREF est l'application informatique de gestion des dossiers des ressortissants étrangers en France, mise en place par le ministère de l'intérieur.

4. Le principe d'estimation des «entrées directes » issues de l'EEE pour les années 1990-1996 figure en annexe 1.

5. Au cours des périodes précédentes, la structure par âge estimée des « entrées différées » était un peu plus jeune. On évaluait ainsi que les moins de 25 ans représentaient les trois quarts des « entrées différées ». Cette différence est à attribuer à l'utilisation, pour les années 1999-2001, de taux d'activité par âge pour estimer les fréquences cumulées d'entrée sur le marché du travail, tandis que nous avions travaillé à partir de données par groupe d'âge quinquennal pour les années antérieures (cf. annexe 2).

\section{RÉSUMÉS}

Chaque année, plusieurs milliers d'étrangers entrent en France afin d'exercer une activité professionnelle. Mais ce ne sont pas les seuls étrangers qui accèdent au marché de l'emploi français. En effet, parmi les étrangers qui entrent chaque année légalement en France à un autre titre que l'exercice d'une activité professionnelle pour une durée au moins égale à un an, certains deviennent actifs l'année même de leur arrivée en France. D'autres diffèrent leur accès au marché de l'emploi les années suivantes. Leur effectif ne faisant l'objet d'aucun dénombrement, il s'est avéré nécessaire de développer une méthode d'estimation destinée à en approcher l'ordre de grandeur. Cette réflexion, initiée en 1996, permet aujourd'hui de disposer d'une série 
statistique couvrant les années 1990-2001, qui précise non seulement les variations quantitatives qu'a connues ce flux depuis 1990, mais également les évolutions de certaines de ses caractéristiques (groupe de nationalités, mode d'accès au marché du travail).

Entries of Foreigners into the French Labour Market (1990-2001). Every year, thousands of foreigners come to France in order to work. However, they are not the only foreigners to enter on the French labour market: among those who, every year, come legally in France for other reasons than working - at least during one whole year - some of them enter on the labour market the very same year they arrived in France. But others do so after several years spent in France. In order to evaluate their numbers, it became necessary to develop an estimation method. Thanks to this method, which dates back to 1996, a statistical series covering the years 1990-2001 is available, which provides information on the quantitative changes in the yearly flows since 1990, but also on some of their feature, such as nationality groups and type of access to the labour market.

Las entradas de extranjeros en el mercado laboral francés (1990-2001). Cada año, miles de extranjeros entran en Francia con el objetivo de ejercer una actividad profesional, mas no son los únicos extranjeros que acceden al mercado del trabajo francés. Entre todos los extranjeros que entran legalmente en Francia cada año por un periodo de al menos doce meses bajo otro estatuto que el de trabajador algunos pasan a ser activos durante dicha estancia. Por el contrario, otros retrasan su acceso al mercado del empleo a los años posteriores. Dado que no existe ningún tipo de contabilización de este fenómeno, ha sido necesario desarrollar un método de estimación significativo. Esta reflexión fue iniciada en 1996 y permite, hoy en día, disponer de una serie estadística que cubre la década 1990-2001. Estos datos recogen las variaciones que estos flujos han sufrido desde 1990, así como las evoluciones de los rasgos que los caracterizan, como por ejemplo el grupo de nacionalidades o el modo de acceso al mercado laboral.

\section{INDEX}

Index géographique : France

Mots-clés : entrée, flux, marché du travail, statistiques

\section{AUTEUR}

\section{JEAN-FRANÇOIS LÉGER}

Chercheur associé au Laboratoire Populations et interdisciplinarité (POPINTER, Université René Descartes - Paris V, 45 rue des Saints-Pères, 75006 Paris) 